\title{
¿LIMITES DEL PENSAMIENTO EXTENDIDO? UNA LECTURA DE LAS CONFERENCIAS SOBRE LA FILOSOFÍA POLÍTICA DE KANT DE HANNAH ARENDT
}

\author{
Francisco Abril \\ Universidad Nacional de Córdoba, Argentina
}

\begin{abstract}
RESUMEN:
En el presente trabajo reconstruyo las reflexiones realizadas por Hannah Arendt en sus Conferencias sobre la filosofía política de Kant. Específicamente, pondré el foco de la atención en la concepción de mentalidad amplia y cómo esta concepción se relaciona con otras ideas que Arendt recupera de la Crítica del juicio y que terminan amalgamándose con su propuesta filosófica. En este punto se suscita un problema que es menester señalar. Se trata de una tensión o contradicción que se produce en las Conferencias entre la idea kantiana de mentalidad amplia y la exhortación que hace la autora a elegir activamente -tanto en política, filosofía y arte, como en la vida en general- las preferencias y las compañías. En el artículo arriesgaré una hipótesis de lectura que podría en principio disolver esa tensión, a saber: que si bien es preciso, en momentos críticos, contar con algún tipo de criterio respecto a qué perspectivas deben ser tomadas en cuenta en virtud de una amplitud de miras y cuáles no -por ejemplo, entre quienes tienen capacidad para juzgar y quienes no la tienen-, pasado dicho momento esos criterios deben revisarse y ponerse en tela de juicio. De lo contrario, se corre el riesgo de desvirtuar la idea kantiana de mentalidad amplía restringiéndola en función de nuestras preferencias.
\end{abstract}

PALABRAS CLAVE: Arendt; Kant; Mentalidad amplía; Juicio; Reflexión moral.

\section{LIMITS OF THE BROAD-MINDED? AN INTERPRETATION OF ARENDT'S}

\footnotetext{
* Facultad de Filosofía y Humanidades (Universidad Nacional de Córdoba, Argentina). Becario doctoral de CONICET. Email: franciscoabril_2@hotmail.com
} 


\title{
LECTURES ON KANT'S POLITICAL PHILOSOPHY
}

\begin{abstract}
:
In this paper I reconstruct the reflections of Hannah Arendt in her Lectures on Kant's Political Philosophy. I'll put the focus of attention specifically in the conception of broad-minded and how this conception relates to other ideas that Arendt recovered from the Critique of Judgment and end up merging with her philosophical proposal. At this point a problem arises that it is necessary to note. It is a contradiction produced in her Lectures between the Kantian idea of broad-minded and the exhortation of the author to actively choose our companies -both in politics, philosophy, art and our lives in general-. In the article I'll risk a hypothesis that would dissolve the contradiction: that while it is necessary, at critical moments, to have some kind of criteria on what perspective should be taken into account under an open mind -for example, the ability to judge-, when the time changes these criteria should be reviewed. Otherwise, we run the risk of restricting the Kantian idea of broad-minded to our preferences.
\end{abstract}

KEYWORDS: Arendt; Kant; Broad-minded; Judgement; Moral reflection.

\section{Introducción}

En el presente trabajo analizo la propuesta que Hannah Arendt elabora en sus Conferencias sobre la filosofía politica de Kant. Estas conferencias fueron dictadas en la New School for Social Research en el curso semestral del otoño de 1970. Arendt muere en el ' 75 con su último libro La vida del espíritu sin concluir. Llegó a redactar dos de las tres partes en las que tenía previsto organizarlo. La primera se tituló "El pensamiento"; la segunda, "La voluntad"; la tercera iba a estar destinada a la problemática kantiana del juicio. La autora sólo pudo escribir el encabezamiento y dos epígrafes correspondientes a esta última parte. Por lo tanto, no pudo darle una conclusión a su estudio dejando un impasse entre el análisis del pensar y el de la voluntad. Las Conferencias son lo más cercano a esta última parte que Arendt dejó como legado a la posteridad. A lo que puede sumársele artículos tales como "El pensar y las reflexiones morales" y "La crisis en la cultura. Su significado social y político", entre otros.

Vale aclarar que, como señala Ronald Beiner (2003, 161 y 162), autor del ensayo interpretativo que acompaña la edición en castellano de las Conferencias, en este momento se producen varios cambios sustantivos en la reflexión de Arendt. En particular, la cuestión del juicio se disocia del 
principio de la acción al que estaba relacionado, como phronesis ${ }^{l}$, en sus trabajos anteriores. Esto lleva incluso a que se le adjudiquen "dos teorías del juicio" (BEINER, 2002, 161): una en la que el juicio y la acción política están estrechamente relacionados entre sí; el actor debe reflexionar de forma extendida sobre sus actos y sus palabras en la escena pública; otra, la de sus últimos textos, en la que el juicio se asocia prioritariamente al espectador, al historiador y al juez. Estos últimos textos, si bien en principio parecen sobre todo de carácter filosófico-epistemológico, están aún fuertemente marcados por el interés en la política y por las experiencias históricas vividas por la autora. El fantasma presente del totalitarismo y el juicio a Eichmann que la motivó a elaborar el concepto de banalidad del mal los recorre transversalmente como su quaestio facti.

Específicamente, me interesa trabajar aquí la concepción de mentalidad amplia y cómo esta concepción se relaciona con otras ideas que Arendt recupera de la Crítica del juicio y que terminan amalgamándose con su propuesta filosófica. En este punto se suscita un problema que quiero señalar y que Beiner no incluye entre las "preguntas críticas" (2002, 229249) que le formula a Arendt en su ensayo. Se trata de una tensión o contradicción que se produce en las Conferencias entre la idea kantiana de mentalidad amplia y la exhortación que hace la autora a elegir activamente tanto en política, filosofía y arte, como en la vida en general- las preferencias y las compañías. Antes de desarrollar este problema, veamos a qué se refieren tanto Kant como Arendt con la primera de las nociones en tensión.

\section{A propósito de la mentalidad amplia}

Para explicar la idea de mentalidad amplia es preciso relacionarla con otros conceptos, quizá el más evidente de los cuales sea el de pluralidad. Kant, según la lectura de Arendt, insiste en la necesidad de que el juicio se construya incorporando diferentes puntos de vista o perspectivas sobre un asunto en particular. No resulta indispensable que estas perspectivas correspondan a la de uno o varios interlocutores reales, sino que pueden ser meramente posibles. En definitiva, de lo que se trata es de analizar una situación, un problema, una cuestión desde distintos enfoques para así ganar

\footnotetext{
${ }^{1}$ Sólo por señalar un pasaje en el que este vínculo aún estaba presente: "Su peculiar ideal [dado en la polis griega, F.A.], su modelo para la aptitud específicamente política está en la phronesis, aquel discernimiento del hombre político (...), que tiene tan poco que ver con la sabiduría que Aristóteles incuso la remarcó como opuesta a la sabiduría de los filósofos. Discernimiento en un contexto político no significa sino obtener y tener presente la mayor panorámica posible sobre las posiciones y puntos de vista desde los que se considera y juzga un estado de cosas. (...) Es en Kant en quien la reencontramos (...). La llama «el modo de pensar más extendido» y la define explícitamente como la capacidad «de pensar desde la posición de cualquier otro»." (ARENDT, 2005, 112)
} 
en comprensión; no es fortuito, por lo tanto, que Kant hablara aquí de pensamiento "ampliado" (ARENDT, 2003, 260).

La idea de mentalidad amplia figura, en el parágrafo 40 de la Crítica del juicio, como la segunda máxima del sensus communis ${ }^{2}$. Esta máxima apela a la capacidad de ponerse en el lugar de los otros (KANT, 2003, 259). Sin embargo, como se aclaró más arriba, no se alude necesariamente a otros reales que, en una discusión real ${ }^{3}$, esgriman argumentos diferentes de los míos. Tanto Kant como Arendt están pensando en una suerte de representación imaginaria de ese otro que, en mi proceso solitario de reflexión, discute conmigo y evalúa si mis opiniones y creencias están justificadas, tienen o no validez. Kant utiliza frecuentemente las metáforas del "ir de visita" (ARENDT, 2002, 84) o de paseo para dar cuenta de este "ponernos en el lugar de los otros".

Este "dos en uno" (ARENDT, 1995, 136), este diálogo en soledad o mejor, para usar la palabra que acuña Arendt, en "solitud" $(2002,112)$ tiene varias consecuencias a destacar. En primer lugar, me sitúa, a la hora de juzgar un asunto particular, más allá de mis condiciones subjetivas, privadas y egoístas. La probabilidad de llegar a un juicio correcto, ya sea en política o en estética, aumenta cuando uno deja atrás estas condiciones subjetivas. Esto quiere decir que, en gran medida, el pensamiento extensivo exige del sujeto que deje de tomarse a sí mismo como punto de referencia. Le exige que deje de considerar correctas sus opiniones por el mero hecho de que sean propias o por falta de imaginación para advertir la multiplicidad de aristas que puede tener una situación o problema. En segundo lugar, el requisito de la mentalidad amplia me obliga a revisar mis creencias, a desmantelar mis prejuicios. Abandonar las condiciones subjetivas e idiosincrásicas es al menos el primer paso que debo dar para librarme de mis creencias infundadas y mis prejuicios personales. En este sentido, el pensamiento extensivo debe entenderse como una de las formas del pensamiento crítico e ilustrado y tiene una función destructiva. Su función es a punto tal destructiva que amenaza con dejarnos sin "barandillas" para pensar y sólo con dudas e interrogantes. Es lo que ocurría con los interlocutores de Sócrates y con él mismo: se veían paralizados por el pez

\footnotetext{
${ }^{2}$ La primera: pensar por uno mismo; la tercera: estar de acuerdo con uno mismo.

3 Una lectura diferente sobre este punto es la de Albrecht Wellmer. En su artículo "Intersubjetividad y razón", este autor insiste en la necesidad de poner el acento en las discusiones reales -así como en los puntos de vista de los otros reales- y no tanto posibles o imaginarias. “(...) ponerse en el lugar de cada uno de los demás es lo mismo que asegurarse que uno no ha pasado por alto ningún argumento válido u objeción. Pero, si no es poniéndonos nosotros mismos en el lugar de cada uno de los demás, ¿cómo podemos asegurarnos de esto? Yo creo que la única respuesta posible es: averiguando lo que los auténticos otros tienen que decir (...) buscando las objeciones reales". (WELLMER, 1988, 251 , las cursivas son nuestras)
} 
"torpedo" destructor de todo" (ARENDT, 2002, 69).

La mentalidad amplia -al igual que el pensamiento en general- obliga a mantenerse equidistante tanto del dogmatismo como del escepticismo. Por un lado, hace evidente el hecho de que no hay creencias definitivas $y$, por ende, deja al descubierto la futilidad de las escuelas filosóficas y cualquier tipo de fundamentalismo. Arendt dirá al respecto que "el pensamiento crítico es, en principio, antiautoritario" (2002, 76). Pero, por otro lado, desconfía del escepticismo y en esto vuelve a tener como referente a Sócrates: hay un vínculo entre reflexión y virtud y, aún cuando este vínculo no pueda defenderse racionalmente de manera satisfactoria, es preciso mantenerlo eludiendo toda clase de nihilismo. Nihilismo que, por su parte, extrae del ejercicio crítico del pensamiento las consecuencias incorrectas: como no hay modo de conocer qué es la virtud, entonces tengo vía libre para vivir de manera licenciosa.

En las Conferencias Arendt recupera dos nociones que serían centrales para la filosofía política (no escrita) de Kant, a saber, la sociabilidad y la comunicabilidad que están supuestas en la facultad de juzgar y, por añadidura, en la máxima de la mentalidad amplia. Son dos nociones que se implican mutuamente la una a la otra. Cuando juzgo esto como bello o feo, bueno o malo, nunca lo hago en el aislamiento. La actividad de juzgar supone siempre una referencia al otro, a una comunidad de hombres. Entiéndase bien: esto no significa que uno se vea obligado a dar a conocer en cada momento y ante cada interlocutor, qué piensa y cómo juzga, sino que el juicio desde su articulación misma supone la posibilidad de ser comunicado y comprendido por otras personas. Julio de Zan, en su artículo dedicado a Arendt, lo explica con claridad:

(...) la comunicabilidad queda definida de tal manera que el propio sujeto, mediante su reflexión, debe poder determinar y juzgar lo que es universalmente comunicable sin necesidad de la comunicación. La definición kantiana precisa por lo tanto que "el gusto es la facultad de juzgar a priori la comunicabilidad de los sentimientos (DE ZAN, 2005, 870).

El principio de publicidad tiene, también, una importancia de primer orden. Se trata de la libertad para comunicar -la "libertad de pluma" (ARENDT, 2002, 44)- a los demás en el espacio público común qué pienso respecto a esta o aquella situación política, o con respecto a esta o aquella pintura, escultura, partitura, etc.; libertad que va unida por definición al juicio y no puede comprenderse por separado. La publicidad es un "principio trascendental" (ARENDT, 2002, 93) y, como dice Ronald Beiner, "pensar en público puede ser constitutivo del pensamiento como tal" $(2002,212)$. La

\footnotetext{
${ }^{4}$ Uno de los nombres que se le daban a Sócrates, junto con el de "comadrona" y el de "tábano" (ARENDT, 1995, 123 y 124).
} 
publicidad, la libertad de pensamiento y expresión debe estar, a su vez, respaldada y garantizada por determinadas instituciones políticas, es decir, por un "régimen de libertad" (DE ZAN, 2005, 871).

El vínculo entre juicio y publicidad tiene varias implicancias. Antes que nada me lleva, no sólo a exponer mis pensamientos y opiniones de forma tal que sean comprensibles por todos, sino que, al hacerlo, deberán pasar por el examen del diálogo público y podrán resistirlo o no. Esta instancia es un requisito insoslayable de todo pensamiento crítico e ilustrado y es la clase de examen a la que Kant quería exponer sus propios trabajos filosóficos; como dice Arendt “(...) él, a diferencia de casi todos los filósofos, lo lamentaba profundamente [tener pocas posibilidades de convertirse en popular, F.A.] y jamás abandonó la esperanza de que fuese posible popularizar su pensamiento" (2002, 77). Esta necesidad de "popularizar su pensamiento", no estaba motivada por afán de reconocimiento, sino porque ésta era la mejor forma de ponerlo a prueba públicamente.

Asimismo, la publicidad y la comunicabilidad de mis ideas implican la existencia de un acuerdo tácito con el otro: que hablamos un mismo lenguaje y que, usándolo, debo ser capaz defender persuasivamente -el Logon didonai de los griegos: dar cuenta, probar, justificar (ARENDT, 2002, 82)- mis afirmaciones y opiniones. Subrayo el término persuasivamente porque, como no es posible alcanzar un conocimiento verdadero en lo que respecta a los asuntos prácticos-públicos, lo que entra en juego es la retórica y la persuasión. Una última implicancia que es preciso señalar refiere a algo que mencioné líneas arriba, a saber, que nuestro pensamiento y nuestras operaciones reflexivas discurren como si se trataran de un diálogo en solitud y, por ende, tienen un carácter discursivo e intersubjetivo.

La mentalidad amplia es una perspectiva equidistante. Es una perspectiva que, para ganar en amplitud, debe mirar las cosas desde afuera y desinteresadamente. Si se quiere, es la perspectiva del "espectador" más que la del actor. Incluso más, la perspectiva del espectador tiene "primacía" (ARENDT, 2002, 122) por sobre la del actor. Lo que le interesa resaltar a Kant y a Arendt no es tanto la importancia del actor ni de su actuación ni de los hechos que se encadenan narrativamente uno tras otro. Lo importante de la Revolución Francesa, por ejemplo, no era sólo quiénes la llevaron a cabo ni cómo, sino que suscitó la atención del resto del mundo y los llevó a reflexionar sobre ella. Dio lugar a que espectadores de todo el mundo ciudadanos de un mundo común, ciudadanos cosmopolitas- dirigieran su mirada a esos acontecimientos, se entusiasmaran con ellos y le buscaran un sentido. No sería forzoso afirmar aquí que para ambos autores la tarea del historiador, en tanto narrador, es equivalente a la del espectador y la historia es aquello susceptible de ser narrado una vez que concluyó -Arendt recurre a las célebres palabras de Hegel: la lechuza de minerva levanta su vuelo 
cuando cae el día $(2002,142)$. Y esta tarea se lleva a cabo aún cuando el progreso de la historia sea infinito y el historiador sea conciente de que en tanto infinito carece de sentido y carece de finalidad y esa conciencia traiga aparejada, casi de forma inexorable, una mirada de hastío y de melancolía que a la larga lo obligará a ponerle punto final a la narración.

Mirada, entonces, de espectador y de historiador. Habría que agregar, y esto es de particular importancia para el problema que señalaré más adelante, se trata también de la perspectiva del juez. Para tener una comprensión ampliada de una situación y para poder dar con una solución imparcial ${ }^{5}$, el juez debe ponerse en el lugar de las personas afectadas, debe atender a lo que ellas dicen y a cómo entienden la situación que de hecho las afecta. Es decir, el juez debe aquí cumplir con la máxima del pensamiento extendido antes de llegar a una sentencia. A su vez, debe mantenerse equidistante, desinteresado y sobre todo no verse afectado en su juicio. El juez trabaja como el poeta ciego: "al cerrar los ojos uno se convierte en espectador imparcial de las cosas visibles, no afectado directamente: el poeta ciego" (ARENDT, 2002, 127, las cursivas son mías). ${ }^{6}$

Tanto el juez, como el historiador y el espectador hacen uso de su facultad de juzgar. Juzgan sobre una situación particular. Y para hacerlo no toman como punto de partida un concepto previamente dado, sino que proceden en sentido contrario: desde el particular hasta la reflexión y/o explicación de carácter general. Esta fijación en lo particular es lo característico del "juicio reflexionante" -en él falta lo universal, debe encontrarse partiendo de un caso concreto- en contraste con el "juicio determinante" -lo universal es, aquí, lo dado a la subsunción-.

Lo llamativo es que tanto Kant como Arendt vinculan fuertemente facultad de juzgar y gusto ${ }^{7}$. El gusto se aplica siempre sobre algo puntual, algo que me gusta o me disgusta, que me parece bello o me despierta "asco" (ARENDT, 2002, 126). Y por eso puedo decir que me gustó esta película o esta obra de teatro o esta o aquella pintura. No pueden rebasarse los límites que impone el caso particular en materia de gusto. Pero lo que resulta

\footnotetext{
${ }^{5}$ Dirá Arendt: "El espectador es imparcial: no se le asigna ningún papel, pues retirarse de toda participación directa para situarse en una posición más allá del juego es una conditio sine qua non de todo juicio", (2002: 105).

${ }^{6}$ Beiner advierte en la importancia que tiene la figura del poeta ciego el desplazamiento que se produce en la filosofía de Arendt: "El acento se desplaza del pensamiento representativo y de la mentalidad amplia de los actores políticos al espectador y al juicio retrospectivo de los historiadores y de los narradores de historias. El poeta ciego, alejado de la acción y, por ello, capaz de una reflexión desinteresada, se convierte ahora en la figura emblemática de la facultad de juzgar". (2002: 161)

${ }^{7}$ La diferencia es que el juicio no está tan implicado o comprometido con el objeto representado como sí lo estaría el gusto. Es lo que ocurría con los espectadores de la revolución francesa. Ellos podían mantener cierta distancia para poder juzgar desinteresadamente -pero de forma entusiasta- sobre lo que estaba ocurriendo. (Véase lo que dice ARENDT, al respecto, 2002: 124).
} 
"llamativo" es que el gusto es un sentido privado, subjetivo -de los más subjetivos que hay, junto con el olfato- y cuando uno piensa en la tarea del juez o del historiado espera -como, por otra parte, señala la máxima de la mentalidad amplia- imparcialidad, no injerencia de las condiciones subjetivas en la actividad de juzgar.

Según Arendt, lo que para Kant viene a resolver este cuello de botella es la noción de sensus communis. Arendt trabaja esta noción en las últimas conferencias, particularmente en la duodécima. Centrándose en el parágrafo 40 de la Crítica del juicio, dirá lo siguiente:

El gusto es ese «sentido comunitario» (gemeinschaftlicher Sinn), y «sentido» quiere decir aquí «un efecto de la mera reflexión sobre el espíritu». Esta reflexión afecta como si se tratase de una sensación, y precisamente de una sensación de gusto, el sentido que discrimina y escoge. «Podríase incluso definir el gusto, como facultad de juzgar aquello que hace generalmente comunicable nuestro sentimiento [como sensación] en una representación [no percepción] dada, sin intervención de un concepto» $(2002,132)$.

En esta definición hay varios de los elementos centrales de la concepción kantiana del sensus communis. En principio, que más que sentido común en su acepción corriente, de lo que se trata es de un "sentido comunitario". Esto es: un sentido, comparable con nuestros órganos sensitivos, compartido por todos los hombres al igual que la vista, el tacto, etc. Como destaca Arendt, se trata de "un sentido adicional -una suerte de capacidad mental añadida (Menschenverstand) - que nos capacita para integrarnos en una comunidad" (2002, 130); Kant lo denomina en el parágrafo cuarenta «entendimiento común humano» $(2003,258)$.

El gusto depende, entonces, del sensus communis. No hay gusto con independencia de este sentido comunitario. Se vuelve problemática la afirmación: "esto me gusta porque me gusta. El gusto es subjetivo, por lo tanto, no me veo obligado a dar ninguna justificación". Arendt -basándose en Kant- podría responder a esto: "el juicio descansa sobre una capacidad que todos compartimos. Tanto el gusto como el sentido común tienen una dimensión intersubjetiva que no puede ignorarse. El hecho de que algo me guste supone esta dimensión así como supone la existencia de otras personas a quienes puedo comunicarles mis gustos y preferencias". El gusto exige lo que la máxima de la mentalidad amplia: que se rebasen las condiciones subjetivas y privadas del juicio y, pese a lo que suele considerarse, gusto e imparcialidad no son palabras que se contraponen entre sí, no están necesariamente reñidas una con otra.

Otro aspecto que puede observarse en la definición citada es que el gusto comporta una elección. Las palabras empleadas son "discrimina y escoge" (ARENDT, 2002, 132). No todo da lo mismo. El sujeto se ve 
obligado a tomar partido por una u otra cosa. El gusto lo saca de la apatía y la indiferencia. Sumado al hecho de que es algo intrínsecamente comunicable por remitir a una representación y, por ende, nos acerca o nos aleja de aquellas personas con las que compartimos o no ciertas preferencias. Antes de entrar en esta temática, central para el problema que busco explicitar, es preciso abrir un breve paréntesis.

Lo que comunico y, en última instancia, aquello que me gusta o desagrada es, no tanto el objeto sensible, como la representación que me hago del mismo. Esta representación es el resultado de la "operación de la imaginación" (ARENDT, 2002, 121 y 124), de la capacidad para evocar mentalmente un objeto que no está siendo aprehendido en el aquí y ahora de mi intuición sensible. Es una re-presentación, una imagen que reconstruyo del objeto en mi subjetividad. Esta imagen interna es lo que afecta y sensibiliza al espíritu. Es lo que suscita la "operación de reflexión" (ARENDT, 2002, 121 y 127) a partir de la cual se evalúa favorable o desfavorablemente el objeto representado y que hace comunicable y comprensible por los otros mis juicios. Lo que se considere agradable o desagradable tendrá más que ver con los resultados de estas operaciones que con una sensación inmediata. Lo que termina por agradarnos no es un objeto bello sino el hecho de que lo consideremos bello -la consideración mismay que esto pueda ser transmitido y compartido por una comunidad. Se trata, entonces, del "efecto de la reflexión sobre el espíritu", de una suerte de sensación mediada reflexivamente que se produce en el espíritu. De ahí la frase de Kant "bello es lo que place en el mero enjuiciamiento" $(2003,272)$ que Arendt retoma diciendo "lo bello es lo que place en la representación, puesto que la imaginación lo ha preparado de forma que yo ahora puedo reflexionar sobre ello" $(2002,124)$.

Cerrando los paréntesis, hay por lo menos dos citas muy sugerentes en las que Arendt insiste en la elección de la compañía. La primera está en la decimotercera conferencia, y surge a partir de una referencia a Cicerón: "Al comunicar los sentimientos, los placeres y goces desinteresados, se expresan preferencias y se eligen compañías. «Preferiría estar equivocado con Platón, que acertado con los pitagóricos»" (ARENDT, 2002, 136, las cursivas son mías). Esta sentencia la explicará más exhaustivamente en el ensayo "La crisis en la cultura: su significado político y social" que se encuentra en el libro Entre el pasado y el futuro:

(...) esta frase puede significar: prefiero equivocarme con la racionalidad platónica antes que «sentir» (sentire) la verdad con la irracionalidad pitagórica, pero esta interpretación no es muy sostenible en vista de la respuesta que se lee en el diálogo: «No me disgustaría equivocarme con un hombre como él» («Ego enim ipse cum eodem isto non invitus erraverim»), donde una vez más el acento está en la persona en cuya compañía se puede errar. Por tanto, parece justificada la primera traducción y la frase significa con toda claridad: es

¿Límites del pensamiento extendido? una lectura de las conferencias sobre la filosofía política de Kant de Hannah Arendt - Francisco Abril 
cuestión de gustos preferir la compañía de Platón y sus pensamientos aun cuando nos lleven a equivocarnos en cuanto a la verdad. (ARENDT, 1996, 237)

Arendt concluye elocuentemente su ensayo diciendo:

\begin{abstract}
En cualquier caso, recordemos lo que los romanos -el primer pueblo que se tomó la cultura en serio tal como lo hacemos nosotros- pensaban que debe ser una persona culta: la que sabe cómo elegir compañía entre los hombres, entre las cosas, entre las ideas, tanto en el presente como en el pasado (1996, 238, las cursivas son nuestras).
\end{abstract}

\title{
¿Una mentalidad amplia limitada?
}

Dicho esto, vuelvo a la formulación del problema que mencioné, sucintamente, al comienzo del trabajo. Si la mentalidad amplia supone la incorporación de la mayor cantidad de perspectivas o puntos de vista (pluralidad) sobre un asunto o conflicto particular, entonces: ¿cómo debe interpretarse el llamado a elegir mis compañías, lo cual supone ciertamente que es preciso dejar de lado a quienes no lo son? ¿Este llamado alude sólo al hombre de acción o también al historiador-espectador? En caso de que lo incluya y que el historiador-espectador se vea por así decir llevado a tomar partido -como dice De Zan: "frente a los hechos políticos la «manera de pensar», o el juicio reflexivo, adquieren el carácter de una toma de partido" (2005, 878)- entonces ¿se lo puede seguir llamando imparcial sin que se desvirtúe el significado de esa palabra?

Habría, en principio, un error en el planteo de este problema. Elegir compañía no quiere decir dejar fuera de la mentalidad amplia a quienes no consideramos nuestros compañeros. Pero, ¿qué sucede con las personas que no ejercen su facultad de juzgar (Eichmann, por ejemplo)? ¿Es necesario incluir su perspectiva si se quiere alcanzar una mentalidad amplia, si se quiere ser testigo imparcial de muchas de las cosas que sucedieron durante la Segunda Guerra Mundial -por ejemplo, la Solución Final-? Por la preocupación que muestra Arendt en comprender a Eichmann, uno se ve tentado a decir que sí. Sin embargo, la caracterización que hace de él es bastante ambivalente: dice de él que era "difícil no sospechar que fuera un payaso" (ARENDT, 1999, p. 37), sin por ello llegar a llamarlo estúpido (ARENDT, 1999, 172). Por otra parte, ¿qué sucedería si en lugar de elegir como ejemplo a Eichmann elegimos a una persona como Carl Schmitt? Arendt respondería: "la cuestión aquí no es que la persona sea o no inteligente -siempre que uno entienda que Carl Schmitt lo era-, ya que una persona inteligente y formada puede carecer de juicio". No obstante, las preguntas que formulo recuerdan la observación de De Zan cuando critica la metáfora kantiana del ir de visita: 
La reiterada metáfora de la "visita" o del "ir de paseo" fuera de sí mismo, me parecen sin embargo demasiado placenteras, esconden el lado intempestivo, desagradable y provocativo de la interpelación del otro. Uno sale de buen grado de visita a la casa de los amigos, de los que son como uno, donde sabe que va a escuchar lo que le gusta oír. Pero no es en estas visitas que se rompe la cáscara, se abren nuevos horizontes y se progresa en una mayor amplitud de pensamiento $(2005,875)$.

Ahora bien, sospecho que la cuestión de fondo es cómo sabe Arendt quiénes ejercitan y quiénes no su facultad de juzgar en situaciones críticas. Entiendo que este problema está relacionado con lo que Albrecht Wellmer denomina críticamente como la existencia de una "mitología del juicio", esto es, una vía a partir de la cual "la facultad de juzgar empieza a aparecer como una facultad un tanto misteriosa de acertar con la verdad cuando no hay contextos de argumentos posibles (...)" (WELLMER, 1996, 327).

A modo de hipótesis de trabajo voy proponer dos ideas: primero, que lo que le permite a Arendt discernir entre unos y otros es su elaboración de dos "tipos ideales" (ARENDT, 1995, 119) con pretensión de "validez ejemplar" (ARENDT, 2002, 141): el de Eichmann, por un lado, y el de Sócrates, por otro. Eichmann es el ejemplo de alguien incapaz de reflexión; Sócrates, lo contrario. Uno era incapaz de hablar, de comunicarse, de pensar allende los clichés que le venían dados por su entorno y siempre obedecía sin importar la racionalidad de la norma o la legitimidad de la autoridad ${ }^{8}$. El otro llevaba la reflexión hasta extremos tales que terminaba por desconfiar de toda afirmación que hicieran él o cualquiera de sus interlocutores; era el gran destructor de prejuicios, de dogmas, de falsas escuelas -por no decir, de toda escuela - y sin embargo eludía en cada momento la tentación del nihilismo y la maldad (no así sus discípulos Alcibíades y Critias); la reflexión crítica -que, respondiendo a un diálogo en soledad, se hacía pública y se sometía a la revisión de los demás- estaba unida a la justicia y al bien, aún cuando no se pudiera dar con una definición satisfactoria de lo que era la justicia y el bien: por eso, para Sócrates, era mejor ser víctima de una injusticia que cometerla. El ejercicio de la reflexión -aquello que no se puede enseñar, sólo ejercer- no está disociado de la virtud, aún cuando se

\footnotetext{
8 Arendt lo describe de este modo: "Cuanto más se le escuchaba, más evidente era que su incapacidad para hablar iba estrechamente unida a su incapacidad para pensar, particularmente, para pensar desde el punto de vista de otra persona. No era posible establecer comunicación con él, no porque mintiera, sino porque estaba rodeado por la más segura de las protecciones contra las palabras y la presencia de otros, y por ende contra la realidad como tal" (1999, p. 34). Algo similar dice en "El pensar y las reflexiones morales": "Clichés, frases hechas, adhesiones a lo convencional, códigos estandarizados de conducta y de expresión cumplen la función socialmente reconocida de protegernos frente a la realidad, es decir, frente a los requerimiento que sobre nuestra atención pensante ejercen todos los acontecimientos y hechos en virtud de su misma existencia” (ARENDT, 1995: $110)$.
} 
carezca de buenos argumentos y nuestro interlocutor (por ejemplo, Callicles en el Gorgias) tenga las de ganar. Eichmann está claramente en las antípodas de esto y sin ser una persona particularmente perversa, sin pertenecer a una "estirpe de demonios", fue capaz de participar en la organización y ejecución de la Solución Final (aún cuando no haya tenido el grado de autoridad que el Estado de Israel pretendió atribuirle cuando lo juzgó).

La segunda idea que propongo como hipótesis es que estos tipos ideales son sólo "andaderas" (ARENDT, 2002, 152) que el juicio le proporciona al pensamiento. En alguna medida tienen sólo un valor práctico -lo que posiblemente llevaría a revisar / matizar la afirmación de que hay una separación entre juicio y acción en los últimos escritos de Arendt-: orientan en situaciones críticas en las que necesitamos coordenadas porque aquellas con las que contábamos se vinieron abajo. E incluso tienen un valor coyuntural. Pasada una situación, no puede dejárselos sin revisar -"una vida sin examen no tiene objeto vivirla" (ARENDT, 1995, 127, las palabras corresponden a una cita de Sócrates que hace la autora). Nos sacan, por un momento siquiera, de la parálisis que produce la mayéutica socrática y lo hacen en situaciones en que es imperativo salir. Pero pasado el momento y a los fines de hacer justicia tanto a la enseñanza de Sócrates como a la mentalidad amplia kantiana, deben mostrar toda su precariedad y dejarse arrastrar, finalmente, por el viento destructivo del pensar.

Entonces, retomando lo dicho, lo que permite a Arendt saber quienes ejercitan o no la facultad de juzgar depende de los dos tipos ideales que ella elabora. Pero, al mismo tiempo, estas construcciones tienen una "validez ejemplar" limitada. Pueden servir como "barandillas" y como coordenadas en momentos de crisis e incertidumbre; pasado ese momento deben ser revisados. Esto se aplica sobre todo al caso de Eichmann. Si bien Arendt se tomó muy en serio el trabajo de comprender la personalidad de este funcionario Nazi, como nadie quizá lo hizo en el momento en que se lo juzgó, muchas veces la tipificación que hace de él corre el riesgo de subestimarlo y presentarlo como un "payaso". Considero que esto es susceptible de revisión crítica en trabajos posteriores sobre la temática. Y ello para eludir una versión confortable de la metáfora del ir de visita, una versión que esconda o ridiculice "el lado intempestivo, desagradable y provocativo de la interpelación del otro” (DE ZAN, 2005, 875).

\section{Obras citadas}

ARENDT,, Hannah. Conferencias sobre la filosofía politica de Kant, Buenos Aires: Paidós, 2003.

ARENDT,, Hannah. De la historia a la acción, Barcelona: Paidós, 1995.

ARENDT, Hannah. Entre el pasado y el futuro, Barcelona: Ediciones Península, 1996 
ARENDT, Hannah. Eichmann en Jerusalén, Barcelona: Lumen, 1999.

ARENDT, Hannah. La vida del espíritu, Buenos Aires: Paidós, 2002.

ARENDT,, Hannah. ¿Qué es la política?, Barcelona: Paidós, 2005.

ARENDT, Hannah. "Verdad y Política", texto extraído de Internet, disponible en: http://estafeta-gabrielpulecio.blogspot.com/2009/01/hannaharendt-verdad-y-politica.html

KANT, Immanuel. Crítica del discernimiento, Madrid: Mínimo Tránsito, 2003.

WELLMER, Albrecht. Finales de partida, Madrid: Frónesis, 1996.

WELLMER, Albrecht. Intersubjetividad y razón. Olivé, León, Racionalidad (comp.). México: Siglo XXI, 1988.

DE ZAN, Julio. "Amplitud de pensamiento y capacidad de juzgar: la lectura de H. Arendt de la Crítica del juicio", Revista Portuguesa de Filosofía, Fasc. 3/4, pp. 863-882, 2005. 